Revista de Comunicación y Salud, 2021, Vol. 11, 1-27

Editado por Cátedra de Comunicación y Salud

ISSN: 2173-1675

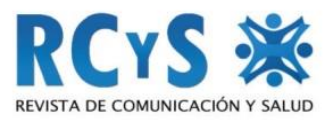

Enviado 21/10/2020

Aprobado 16/02/2021

Publicado 19/04/2021

\title{
INTERÉS Y UTILIZACIÓN DE PELÍCULAS Y SERIES TELEVISIVAS PARA LA EDUCACIÓN PARA LA SALUD EN LA ESCUELA SECUNDARIA
}

\author{
Interest and use of films and TV series for health education in high school \\ Carla Garrido Pérez \\ Universidad Pompeu Fabra. España. \\ carla.garrido02@estudiant.upf.edu
}

\author{
Irene Cambra Badii ${ }^{1}$ \\ Universidad de Vic - Universidad Central de Catalunya. España. \\ irene.cambra@uvic.cat \\ Mar Carrió Llach \\ Universidad Pompeu Fabra. España. \\ mar.carrio@upf.edu
}

\section{Cómo citar el artículo}

Garrido Pérez, C., Cambra Badii, I. y Carrió Llach, M. (2021) Interés y utilización de películas y series televisivas para la educación para la salud en la escuela secundaria. Revista de Comunicación y Salud, 11, 1-27. https://doi.org/10.35669/rcys.2021.11.e266

\section{Resumen}

La educación para la salud tiene un papel clave en la adquisición de las habilidades necesarias para tomar decisiones que afectan a la salud personal o comunitaria. Por esta razón se debería garantizar en la educación obligatoria. Teniendo en cuenta que las películas y series pueden facilitar el aprendizaje de conceptos complejos, se diseñó un cuestionario para identificar los filmes relacionados con las ciencias de la salud que ven los estudiantes de secundaria de manera voluntaria y explorar su uso en las aulas para

\footnotetext{
${ }^{1}$ Irene Cambra Badii: Investigadora en la cátedra de Bioética de la Universitat de Vic - Universitat Central de Catalunya y en el Grupo de Investigación en Educación en Ciencias de la Salud (GRECS) en el Departamento de Ciencias Experimentales y de la Salud de la Universitat Pompeu Fabra, Barcelona, España.
} 
Interés y utilización de películas y series televisivas para la educación para la salud en la escuela secundaria

trabajar la alfabetización en salud. Participaron 237 estudiantes de España. El 54,85\% afirmó que suele ver estos filmes en su vida cotidiana, siendo las series médicas las más vistas; sin embargo, el $57,38 \%$ indicó que no se utilizan estos recursos en las clases. El estudio sugiere un uso potencial de los materiales audiovisuales para trabajar los objetivos de educación para la salud, aunque debe ser fomentado sistemáticamente.

Palabras clave: cine, educación y salud, educación media, educación para la salud, aprendizaje activo, materiales audiovisuales, materiales didácticos

\begin{abstract}
Health education plays a key role in acquiring the skills necessary to make decisions affecting personal or community health and should be guaranteed in compulsory education. Since films and series can facilitate the learning of complex concepts, a questionnaire was designed to inquire whether secondary school students view films related to health sciences on a voluntary basis, and whether they are used in classrooms to work key concepts of health literacy. 237 students from Spain have participated. $54.85 \%$ stated that they usually view these films in their daily lives, with medical series being the most watched; however, $57.38 \%$ indicated that these resources are not used in the classroom. The study suggests a potential use of films and series to work on health education goals, although it should be systematically encouraged.
\end{abstract}

Keywords: Cinema, education and health, secondary education, health education, active learning, audiovisual materials, teaching materials

\title{
1. INTRODUCCIÓN
}

El cuidado de la salud implica una serie de representaciones y prácticas para la promoción de la salud y la prevención de la enfermedad. Aunque podría parecer que tenemos a nuestro alcance toda la información necesaria para cuidar nuestra salud, es necesario tener herramientas para que podamos entender, procesar y aplicar estos recursos de manera productiva (Romero y Ruiz Cabello, 2016).

Es importante que los aprendizajes sobre la salud se den durante la edad infantil y juvenil, ya que este periodo corresponde al de mayor aprendizaje y adquisición de los principales hábitos saludables (Gavidia Catalán et al., 2019). Asimismo, los comportamientos sanitarios establecidos durante la adolescencia tienden a perdurar en la edad adulta (Bruselius-Jensen et al., 2017; Manganello, 2008).

Por este motivo es necesario establecer y promover los conocimientos que mejoran la salud de la ciudadanía, varias organizaciones han definido estándares de educación para la salud (Nutbeam et al., 2018), entre los que destacan -por ser muy completos- los propuestos en Estados Unidos por el Centro para el Control y la Prevención de Enfermedades ("Centers for Disease Control and Prevention") (CDC, 2004). Éstos proporcionan un marco conceptual para el profesorado y los responsables educativos 
Interés y utilización de películas y series televisivas para la educación para la salud en la escuela secundaria

para trabajar de manera progresiva los objetivos y las expectativas de educación para la salud en las diferentes etapas educativas, desde primaria hasta bachillerato. Los estándares incluyen: 1) comprender conceptos relacionados con la promoción de la salud y la prevención de enfermedades, 2) analizar la influencia de la familia, los compañeros, el bagaje cultural, los medios de comunicación, la tecnología y otros factores en los comportamientos relacionados con la salud, 3) acceder a información, productos y servicios fiables para la mejora de la salud, 4) comunicarse de forma efectiva en relación a la salud, 5) tomar decisiones saludables, 6) establecer objetivos para mejorar la salud, 7) desarrollar hábitos saludables y 8) fomentar la salud personal, familiar y comunitaria.

En España, la educación secundaria incluye el nivel de secundaria obligatoria (ESO, 4 cursos, desde los 12 hasta los 16 años) y bachillerato (2 cursos, desde los 16 hasta los 18 años). Su currículo oficial está orientado a la adquisición de competencias, pero éste no incluye una competencia específica de salud ni una asignatura donde desarrollarla. Se puede integrar en otras competencias básicas como la de "conocimiento e interacción con el mundo físico" o "la social y ciudadana" (BOE, Real Decreto 126/2014; BOE, Real Decreto 1105/2014) y trabajar de manera transversal en diferentes asignaturas como biología, educación física o ética y ciudadanía. De todas formas, el hecho de que no esté concretada y definida en el currículo, dificulta el desarrollo de la educación para la salud que deberían adquirir los jóvenes en su etapa escolar. Afortunadamente, existen propuestas recientes de sobre desarrollar las competencias en salud durante la educación obligatoria que pueden servir de referencia para planificar actividades de educación para la salud (Gavidia et al., 2019).

Por otra parte, las propuestas pedagógicas sobre educación para la salud en la escuela secundaria deben estar centradas en estrategias interdisciplinarias y con una visión integral que permita a los estudiantes considerar su contexto familiar, comunitario y social (Rodríguez Torres et al., 2017; Machado, Oliveira y Manica, 2013) y a su vez promover el desarrollo de conocimientos, habilidades y destrezas para el autocuidado de la salud y la prevención de conductas de riesgo (De Lellis et al., 2014).

Resulta una vía de acción para la promoción de la salud, y engloba las intervenciones y actividades educativas encaminadas a mejorar la alfabetización en salud de las personas (Gavidia et al., 2019). Este proceso es definido como:

la alfabetización general engloba las motivaciones, los conocimientos y las competencias de las personas para acceder, entender, evaluar y aplicar la información sobre la salud en la toma de decisiones sobre la atención y el cuidado sanitario, la prevención de enfermedades y la promoción de la salud para mantener y mejorar la calidad de vida a lo largo de ésta (Sørensen et al., 2012, p. 3).

Diversos estudios establecen que la escuela juega un papel clave a la hora de promover la alfabetización en salud (Wager et al., 2020; Salvador y Suelves, 2009; OMS, 1990) y que sirve para mejorar la salud individual y comunitaria (OMS, 1986). Asimismo, otras evidencias demuestran que la alfabetización en salud se puede desarrollar con éxito en las aulas (Bruselius-Jensen, 2017; Nutbeam, 2000). 
Interés y utilización de películas y series televisivas para la educación para la salud en la escuela secundaria

\subsection{El cine y las series en la educación para la salud}

El cine siempre ha tratado temas de salud en tanto que refleja y a su vez produce la cultura y las representaciones de una determinada sociedad (Brigidi, 2016; ÚbedaCarulla, 2016). Las películas ofrecen oportunidades para que revisemos las representaciones sobre las ciencias de la salud -y de las personas que se dedican a la ciencia, médicos, científicos, etc.- a la vez que facilitan la reflexión y el debate sobre ellas (Petit y Solbes, 2012, 2015, 2016). Desde los inicios del cine, se han hecho películas sobre hospitales, enfermedades, investigación con seres humanos. Por esta razón se han utilizado en contextos educativos de escuelas secundarias y universidades (D'Ottavio et al., 2019; Farré 2013).

Las imágenes y las historias relatadas en el cine y las series son elementos disparadores que generan entusiasmo en la audiencia, a la vez que amplían el conocimiento conceptual y fomentan las capacidades críticas. De hecho, distintas investigaciones destacan su carácter motivador, y las facilidades que ofrecen como herramientas educativas, no solo por su llegada a un gran número de personas, sino también por la posibilidad de exposición de los núcleos más interesantes (o controvertidos) del conocimiento (García Borrás 2009; Mateus 2017; Petit y Solbes 2012).

Numerosos estudios han demostrado que la utilización de películas comerciales en las aulas es favorable, mostrando un aprendizaje que proporciona la capacidad para analizar problemas críticamente, razonar las situaciones científicas desde varios puntos de vista, así como la facilidad para construir y consolidar nuevos conocimientos (Arroio, 2010; Baños y Pérez, 2005). Además, el cine supone un impacto sensorial y emocional que implica un refuerzo educativo y facilita la memorización y la asociación de conceptos complejos (Fresnadillo Martínez et al., 2005).

Sin embargo, la mayor parte de los estudios previos se centran en la enseñanza universitaria de las ciencias de la salud (Icart Isern, Garrido-Aguilar, Miguel-Cañas, 2013; Baños y Bosch, 2015) y no se encontraron estudios que relacionen directamente el uso de estos recursos con temas de educación para la salud y alfabetización en salud en la escuela secundaria.

Por otra parte, actualmente las series son consideradas como el reemplazo del cine para las grandes audiencias. Diariamente, millones de personas siguen desde la pantalla de su teléfono móvil, tablet o notebook series de distintos géneros y formatos (desde la tradicional sitcom hasta miniseries de escasos episodios, con un formato similar al cinematográfico) (Cambra Badii y Baños, 2020; Lobato, 2018; Correa Romero et al., 2015). En una investigación reciente realizada con estudiantes de ciencias de la salud, las series son el formato audiovisual preferido (Cambra Badii et al., 2020).

Dentro del universo de las series, las que tratan cuestiones médicas son un producto muy consumido y difundido, sobre todo entre estudiantes de ciencias de la salud (Williams, Re y Ozakinci, 2014; Weaver y Wilson, 2011; Czarny 2008). Estas series tienen como protagonistas a profesionales de la salud en la atención de diferentes

Revista de Comunicación y Salud, 2021, Vol. 11, 1-27 
Interés y utilización de películas y series televisivas para la educación para la salud en la escuela secundaria

servicios hospitalarios: cirugía, obstetricia, emergencias, etc. Los factores que motivan a los estudiantes a mirar series de temas médicos son principalmente entretenimiento e información sobre aspectos médicos, lo cual concuerda con una de las definiciones de las series médicas: una herramienta de entretenimiento-educación (Law et al., 2015; O'Connor, 1998).

Aunque las películas y series sobre temas de salud están presentes desde hace tiempo, recientemente ha surgido un cambio respecto a la visión de la figura de los médicos/as (Cambra Badii, Guardiola y Baños 2020; Tapper, 2010; Chory-Assad y Tamborini 2001) y científicos/as (Kirby, 2017). Antes, estos personajes podían ser más 'ficticios' donde se interpretaban como héroes en el caso de los primeros, o como locos y malvados en el caso de los segundos. Actualmente se tiende a dar una visión más realista y objetiva, mostrando tanto las virtudes como los errores y las dificultades o no que tienen a la hora de realizar su trabajo (Cambra Badii, 2020).

Teniendo en cuenta que el uso de los filmes y series muestran resultados muy efectivos en la educación científica y que la figura del profesor es importante ante el análisis de estos recursos para ayudar a entender el contenido científico en el contexto real (Arroio 2010), se podría deducir que las series y películas relacionadas con las ciencias de salud que miran los adolescentes pueden tener un uso potencial para trabajar los objetivos de educación para la salud en las clases de educación secundaria. Por tanto, los objetivos de este trabajo son 1) identificar qué películas y series relacionadas con ciencias de la salud ven los estudiantes de secundaria de España, 2) identificar qué objetivos de educación para la salud se tratan en estas películas y series y, por último, 3) saber qué uso se hace actualmente de estos recursos audiovisuales en las aulas.

\section{METODOLOGÍA}

\subsection{Diseño}

Esta investigación puede enmarcarse dentro de la investigación educativa (McMillan y Schumacher, 2005) dado que busca aportar conocimiento sobre la posible utilización de películas y series para la educación para la salud en escuelas de educación secundaria. El diseño, de carácter descriptivo, sigue las bases de la metodología cualitativa, entendiendo la importancia de la perspectiva de los informantes (Creswell, 1998; Vasilachis de Gialdino, 2006). Para la consecución de los objetivos, se utilizan métodos cuantitativos y cualitativos. Los primeros permiten describir los perfiles sociodemográficos de los participantes de la investigación y analizar en términos de frecuencias las variables de visionado de películas y series sobre ciencias de la salud, los objetivos de educación para la salud que se articulan con estas películas, y su uso en la escuela secundaria. Los métodos cualitativos permiten categorizar las películas y series más identificadas por los estudiantes según los temas principales de sus narrativas y analizar todos los datos en su conjunto, con el objetivo de explorar sus finalidades didácticas.

\subsection{Participantes}

Revista de Comunicación y Salud, 2021, Vol. 11, 1-27 
Interés y utilización de películas y series televisivas para la educación para la salud en la escuela secundaria

En esta investigación participaron estudiantes de educación secundaria de España (12-18 años). La muestra está compuesta por 237 adolescentes estudiantes de la ESO (Educación Secundaria Obligatoria) o Bachillerato que contestaron el cuestionario Google Forms difundido en línea a través de contactos y las redes sociales (Facebook, Instagram, Twitter y WhatsApp) durante la segunda quincena del mes de mayo de 2020 (Tabla 1).

Tabla 1. Representación de la muestra

\begin{tabular}{|c|c|}
\hline \multicolumn{2}{|c|}{ Género } \\
\hline Mujer & $160(67,51 \%)$ \\
\hline Hombre & $76(32,07 \%)$ \\
\hline Otros & $1(0,42 \%)$ \\
\hline \multicolumn{2}{|c|}{ Curso } \\
\hline 1ํ ESO (12-13 años) & $23(9,70 \%)$ \\
\hline 2ํㅡ ESO (13-14 años) & $14(5,91 \%)$ \\
\hline 3ำ ESO (14-15 años) & $25(10,55 \%)$ \\
\hline 4ํㅡㄹ ESO (15-16 años) & $53(22,36 \%)$ \\
\hline $1^{\circ}$ Bachillerato (16-17 años) & $64(27 \%)$ \\
\hline $2^{\circ}$ Bachillerato (17-18 años) & $58(24,47 \%)$ \\
\hline \multicolumn{2}{|c|}{ Comunidad Autónoma } \\
\hline Cataluña & $182(76,79 \%)$ \\
\hline Otros & $55(23,21 \%)$ \\
\hline
\end{tabular}

Fuente: elaboración propia

\subsection{Técnicas e instrumentos de recolección de datos}

Para la recogida de datos se ha diseñado un cuestionario en línea Google forms (Anexo 1). Este se ha elaborado en castellano con la finalidad de llegar a estudiantes de toda España. Se ha dividido en tres dimensiones donde se analizan diferentes aspectos necesarios para alcanzar los objetivos del trabajo (Tabla 2). 
Interés y utilización de películas y series televisivas para la educación para la salud en la escuela secundaria

Tabla 2. Diseño del cuestionario: dimensiones, datos recogidos y tipología de preguntas

\begin{tabular}{|c|c|c|}
\hline $\begin{array}{l}\text { Dimensiones del } \\
\text { cuestionario }\end{array}$ & Datos recogidos & $\begin{array}{l}\text { Tipología de } \\
\text { preguntas }\end{array}$ \\
\hline $\begin{array}{l}\text { 1. Perfil de los } \\
\text { estudiantes }\end{array}$ & Características personales & Elección múltiple \\
\hline \multirow{3}{*}{$\begin{array}{l}2 . \quad \text { Impacto } \\
\text { películas y series en la vida } \\
\text { cotidiana de los } \\
\text { estudiantes }\end{array}$} & $\begin{array}{l}\text { Películas y series relacionados con la salud } \\
\text { que visualizan los estudiantes de manera } \\
\text { voluntaria }\end{array}$ & Abierta \\
\hline & $\begin{array}{l}\text { Motivación para el aprendizaje de las } \\
\text { ciencias de la salud que generan estos } \\
\text { materiales audiovisuales }\end{array}$ & \multirow{2}{*}{$\begin{array}{l}\text { Escala de Liker } \\
\text { de } 1-5 \text { siendo } 1 \\
\text { "totalmente en } \\
\text { desacuerdo" y } 5 \\
\text { "totalmente de } \\
\text { acuerdo" }\end{array}$} \\
\hline & $\begin{array}{l}\text { Objetivos de educación para la salud que se } \\
\text { tratan en los materiales audiovisuales que } \\
\text { ven los estudiantes. Las preguntas se } \\
\text { basan en los estándares de salud recogidos } \\
\text { en el documento "National Health Education } \\
\text { Standards" (CDC, 2004). }\end{array}$ & \\
\hline \multirow{4}{*}{$\begin{array}{l}\text { 3. Uso de películas y } \\
\text { series en el ámbito } \\
\text { académico }\end{array}$} & $\begin{array}{l}\text { Uso actual de los materiales audiovisuales } \\
\text { en las aulas de educación secundaria }\end{array}$ & Dicotómica (sí/no) \\
\hline & Finalidad didáctica de su uso & \multirow{3}{*}{$\begin{array}{l}\text { Escala de Likert } \\
\text { de } 1-5 \text { siendo } 1 \\
\text { "totalmente en } \\
\text { desacuerdo" y } 5 \\
\text { "totalmente de } \\
\text { acuerdo" }\end{array}$} \\
\hline & $\begin{array}{l}\text { Factores motivacionales del uso de los } \\
\text { materiales audiovisuales en la docencia }\end{array}$ & \\
\hline & $\begin{array}{l}\text { Uso actual de los materiales audiovisuales } \\
\text { para tratar los objetivos de educación para } \\
\text { la salud, basados en el documento } \\
\text { "National Health Education Standards" } \\
\text { (CDC, 2004). }\end{array}$ & \\
\hline
\end{tabular}

Fuente: elaboración propia

\subsection{Análisis de datos}

Para realizar el análisis descriptivo de manera íntegra se ha creado un archivo en Microsoft Excel, donde se han exportado todos los datos obtenidos del cuestionario para hacer un recuento y, posteriormente, poder ser analizados mediante tablas y gráficas. 
Interés y utilización de películas y series televisivas para la educación para la salud en la escuela secundaria

\subsubsection{Análisis cuantitativo}

Para el análisis de las preguntas valoradas con la escala de Likert, se ha calculado tanto la media aritmética $(\square)$ por pregunta, como la desviación típica $(\sigma)$ de estos datos.

También se ha contemplado el porcentaje de las puntuaciones del 1 al 5 de cada respuesta. Respecto a las preguntas de respuesta dicotómica, se ha realizado el análisis a partir de porcentajes.

\subsubsection{Análisis cualitativo}

Para analizar y realizar el recuento sobre qué tipo de películas y series relacionadas con las ciencias de la salud son visualizadas entre los estudiantes, se han categorizado las respuestas de la pregunta abierta "Cita las películas o series que recuerdes haber visto recientemente sobre el ámbito de las ciencias de la salud" de manera inductiva y en función de la temática de los filmes, siempre teniendo en cuenta que los temas de salud formen parte de la trama central.

Para este trabajo, se diseñó una ficha de recogida de datos y dos investigadoras identificaron las distintas categorías. Luego se mantuvieron dos sesiones de revisión de las categorías propuestas y unificación de las categorías (con la tercera investigadora) para garantizar la validez, confiabilidad y reproducibilidad (Krippendorf, 1997).

Se han identificado las siguientes categorías: (i) Evolución, clonación, ingeniería genética y experimentación con humanos, (ii) Epidemias y plagas, (iii) Hospitales, (iv) Enfermedades concretas, y (v) Educación sexual, drogas y adolescentes.

Con todas las respuestas obtenidas, se han categorizado las series y películas identificadas, así como la frecuencia con que se ha mencionado cada una y el número total de menciones por categoría.

Se han descartado 51 menciones de películas y series (12,56\% del total) por ser respuestas no significativas, ya sea porque los materiales audiovisuales no estaban relacionados con temas de salud o porque no se referían a recursos de ficción.

\subsection{Implicaciones éticas}

La participación ha sido totalmente voluntaria y anónima.

Antes de comenzar el cuestionario, se informó a los participantes de los objetivos de la investigación y de su derecho a negarse a participar o abandonar el cuestionario en cualquier momento. Los participantes dieron su consentimiento para participar en el estudio al completar y enviar los cuestionarios de forma anónima. El estudio se realizó de acuerdo con los principios de la Declaración de Helsinki, que garantiza el resguardo de los individuos mediante el consentimiento informado y la confidencialidad de sus respuestas al estudio. 
Interés y utilización de películas y series televisivas para la educación para la salud en la escuela secundaria

\section{RESULTADOS}

\subsection{Impacto de las películas y series en la vida cotidiana}

Después de analizar el cuestionario, obtenemos que, de los 237 estudiantes encuestados, 130 refieren que suelen mirar películas y series relacionadas con el ámbito de las ciencias de la salud (54,85\%). Por otro lado, 107 responden que no suelen mirar recursos de este tipo $(45,15 \%)$.

Cuando se pregunta qué películas o series recuerdan haber visto recientemente sobre este ámbito, hay variedad de respuestas. Entendiendo como mención cada vez que un alumno diferente nombra una película o serie, se han obtenido 406 menciones útiles de 86 materiales audiovisuales diferentes ( 35 series y 51 películas). Estos datos se han categorizado en las categorías descritas en la Metodología. En la Tabla 3 se presentan por orden de más a menos mencionadas y la frecuencia y porcentaje de mención de cada categoría.

Tabla 3. Materiales audiovisuales sobre salud que los estudiantes visualizan de manera voluntaria

\begin{tabular}{|c|c|c|c|}
\hline Categoría & Audiovisuales más mencionados & $\begin{array}{r}\text { Material } \\
\text { audiovisual }\end{array}$ & $\begin{array}{c}\text { Frecuencia } \\
\text { y porcentaje } \\
\text { de mención } \\
\text { de la } \\
\text { categoría }\end{array}$ \\
\hline \multirow{8}{*}{ Hospitales } & $\begin{array}{c}\text { The Good Doctor (ABC, Estados } \\
\text { Unidos, 2017-) }\end{array}$ & Serie & \multirow{8}{*}{$\begin{array}{c}226 \\
(55,67 \%)\end{array}$} \\
\hline & $\begin{array}{c}\text { Grey's Anatomy (ABC, Estados } \\
\text { Unidos, 2005-) }\end{array}$ & Serie & \\
\hline & $\begin{array}{l}\text { House MD (Fox, Estados Unidos, } \\
\text { 2004-2012) }\end{array}$ & Serie & \\
\hline & $\begin{array}{l}\text { Polseres Vermelles (TV3, España, } \\
\text { 2012-2013) }\end{array}$ & Serie & \\
\hline & $\begin{array}{c}\text { Centro Médico (Televisión Española, } \\
\text { España, 2015-2019) }\end{array}$ & Serie & \\
\hline & $\begin{array}{l}\text { Hospital Central (Mediaset, España, } \\
\qquad 2000-2012)\end{array}$ & Serie & \\
\hline & $\begin{array}{c}\text { Chicago Med (NBC, Estados Unidos, } \\
\text { 2015-) }\end{array}$ & Serie & \\
\hline & $\begin{array}{l}\text { The Resident (Fox, Estados Unidos, } \\
\text { 2018-) }\end{array}$ & Serie & \\
\hline
\end{tabular}

Revista de Comunicación y Salud, 2021, Vol. 11, 1-27 
Interés y utilización de películas y series televisivas para la educación para la salud en la escuela secundaria

\begin{tabular}{|c|c|c|c|}
\hline & $\begin{array}{l}\text { Der Medicus (UFA Cinema, } \\
\text { Alemania, 2013) }\end{array}$ & Película & \\
\hline \multirow{9}{*}{$\begin{array}{l}\text { Evolución, } \\
\text { clonación, } \\
\text { ingeniería } \\
\text { genética y } \\
\text { experimentación } \\
\text { con humanos }\end{array}$} & $\begin{array}{c}\text { Gattaca (Columbia Pictures, Estados } \\
\text { Unidos, 1997) }\end{array}$ & Película & \multirow{9}{*}{$\begin{array}{c}100 \\
(24,63 \%)\end{array}$} \\
\hline & $\begin{array}{l}\text { The island (Parkes/MacDonald, } \\
\text { Estados Unidos, 2005) }\end{array}$ & Película & \\
\hline & $\begin{array}{l}\text { Stranger Things (Netflix, Estados } \\
\text { Unidos, 2016-) }\end{array}$ & Serie & \\
\hline & $\begin{array}{l}\text { Orphan Black (BBC, Estados Unidos, } \\
\text { 2013-2017) }\end{array}$ & Serie & \\
\hline & $\begin{array}{c}\text { The } 100 \text { (The CW, Estados Unidos, } \\
\text { 2014-) }\end{array}$ & Serie & \\
\hline & Lucy (Europacorp, Francia, 2014) & Película & \\
\hline & $\begin{array}{l}\text { Jurassic World (Legendary Pictures, } \\
\text { Estados Unidos, 2015) }\end{array}$ & Película & \\
\hline & $\begin{array}{l}\text { Jurassic Park (Amblin Entertainment, } \\
\text { Estados Unidos, 1993) }\end{array}$ & Película & \\
\hline & $\begin{array}{l}\text { Gemini Man (Jerry Bruckheimer } \\
\text { Films, Estados Unidos, 2019) }\end{array}$ & Película & \\
\hline \multirow{7}{*}{$\begin{array}{l}\text { Epidemias y } \\
\text { plagas }\end{array}$} & $\begin{array}{l}\text { The Flu (iFilm Corp., Corea del Sur, } \\
\text { 2013) }\end{array}$ & Película & \multirow{7}{*}{$\begin{array}{c}48 \\
(11,82 \%)\end{array}$} \\
\hline & $\begin{array}{c}\text { Contagion (Warner Bros, Estados } \\
\text { Unidos, 2011) }\end{array}$ & Película & \\
\hline & $\begin{array}{c}\text { World War Z (Skydance Productions, } \\
\text { Reino Unido, 2013) }\end{array}$ & Película & \\
\hline & The Rain (Netflix, Dinamarca, 2018-) & Serie & \\
\hline & $\begin{array}{l}\text { Resident Evil (Netflix, Estados } \\
\text { Unidos, 2020-) }\end{array}$ & Serie & \\
\hline & $\begin{array}{c}\text { Twelve Monkeys (Universal Pictures, } \\
\text { Estados Unidos, 1995) }\end{array}$ & Película & \\
\hline & $\begin{array}{l}\text { I Am Legend (Warner Bros, Estados } \\
\text { Unidos, 2007) }\end{array}$ & Película & \\
\hline \multirow{2}{*}{$\begin{array}{l}\text { Enfermedades } \\
\text { concretas }\end{array}$} & $\begin{array}{c}\text { Five feet apart (CBS Films, Estados } \\
\text { Unidos, 2019) }\end{array}$ & Película & \multirow{2}{*}{$23(5,67 \%)$} \\
\hline & $\begin{array}{l}\text { Brain on fire (Broad Green Pictures, } \\
\text { Estados Unidos, 2016) }\end{array}$ & Película & \\
\hline
\end{tabular}


Interés y utilización de películas y series televisivas para la educación para la salud en la escuela secundaria

\begin{tabular}{|c|c|c|c|}
\hline & $\begin{array}{c}\text { To the bone (Netflix, Estados Unidos, } \\
\text { 2017) }\end{array}$ & Película & \\
\hline \multirow{2}{*}{$\begin{array}{c}\text { Educación } \\
\text { sexual, drogas y } \\
\text { adolescentes }\end{array}$} & $\begin{array}{c}\text { Sex Education (Netflix, Reino Unido, } \\
2019-)\end{array}$ & Serie & \multirow{2}{*}{$9(2,22 \%)$} \\
\cline { 2 - 3 } & $\begin{array}{c}\text { Euphoria (HBO, Estados Unidos, } \\
2019-)\end{array}$ & Serie & \\
\hline
\end{tabular}

Fuente: elaboración propia

Se observa una elevada tendencia hacia las series médicas, dramas que transcurren en hospitales, destacando la serie The Good Doctor con 75 respuestas y Grey's Anatomy con 46. La siguiente categoría más destacada es la de Evolución, clonación, ingeniería genética y experimentación con seres humanos, donde destacan las películas Gattaca y The Island con 19 respuestas la primera y 10 la segunda. Cabe mencionar que a pesar de tener más menciones en el ámbito de hospitales, se han obtenido 22 películas/series diferentes, mientras que en la categoría de evolución, clonación, etc. se han identificado 33 diferentes.

Otra de las categorías identificadas es la de epidemias y plagas, donde se han obtenido 13 películas y series diferentes. La película que más resalta es la coreana The Flu, con un total de 13 menciones. Por último, categorías más minoritarias son las de Enfermedades concretas y Educación sexual, drogas y adolescentes.

Cuando se pregunta si estos recursos provocan una motivación hacia temas relacionados con las ciencias de la salud, la media $(\square)$ de las puntuaciones de la escala de Likert es de 3,46 sobre 5 con una desviación estándar $(\sigma)$ de 1,09, siendo un $54,01 \%$ de los estudiantes que están de acuerdo o muy de acuerdo con esta afirmación.

\subsection{Identificación de los objetivos de educación para la salud que tratan}

Después de identificar qué películas y series relacionadas con las ciencias de la salud miran los estudiantes, se analiza qué temas recuerdan que muestran relacionados con la salud (Gráfico 1). 
Interés y utilización de películas y series televisivas para la educación para la salud en la escuela secundaria

A)

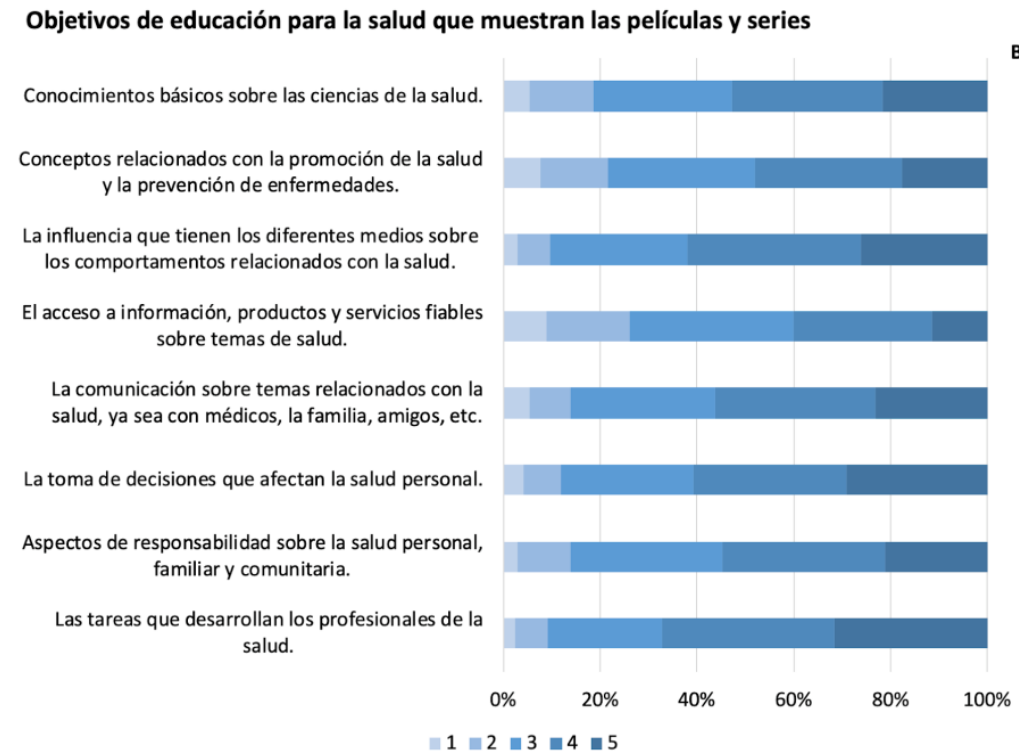

B)
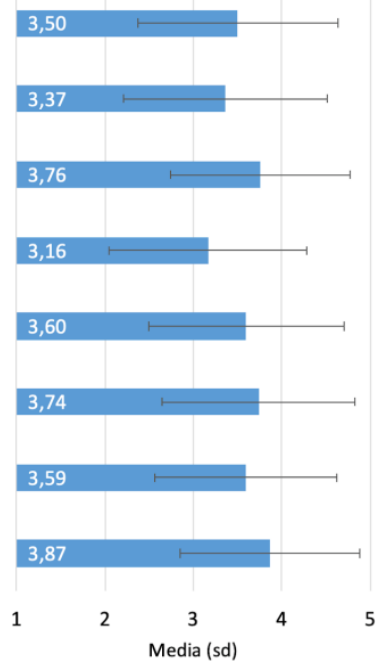

Gráfico 1. Objetivos de educación para la salud que se muestran en las películas y series que los estudiantes suelen mirar de manera voluntaria $(N=237)$. 1A) porcentaje de menciones, 1B) media de las respuestas con la desviación estándar (sd) de cada ítem.

Fuente: elaboración propia

El $52,74 \%$ de los encuestados responden estar de acuerdo o muy de acuerdo con que se muestran conocimientos básicos sobre ciencias de la salud, obteniendo una media de 3,50 sobre $5(\sigma=1,13)$ en la escala de Likert. Respecto a si consideran que estos materiales audiovisuales presentan conceptos relacionados con la promoción de la salud y la prevención de enfermedades se obtiene una media de acuerdo de 3,37 sobre 5 $(\sigma=1,15)$. Si se preguntan conceptos como la influencia que tiene la familia, los compañeros o los medios de comunicación o sobre el acceso a información y servicios fiables respecto a comportamientos relacionados con la salud, obtenemos una media de 3,76 sobre $5(\sigma=1,01)$ y de $3,16(\sigma=1,12)$, respectivamente.

El $56,12 \%$ de los estudiantes está de acuerdo o muy de acuerdo con que, en estos recursos, se muestra la comunicación, ya sea con médicos, familiares o amigos sobre temas relacionados con la salud $(\square=3,60 ; \sigma=1,10)$. También aparecen aspectos relacionados con la toma de decisiones que afectan a la salud personal, con el $60,76 \%$ $(\square=3,74 ; \sigma=1,09)$ de acuerdo o muy de acuerdo; así como de responsabilidad sobre salud personal, familiar y comunitaria, con el $54,85 \%(\square=3,59 ; \sigma=1,03)$. Por último, un $67,09 \%$ ( $\square=3,87 ; \sigma=1,02)$ está de acuerdo o muy de acuerdo en que en éstas películas y/o series, se muestran las tareas que desarrollan los profesionales de la salud, siendo un $31,65 \%$ los que están muy de acuerdo. 
Interés y utilización de películas y series televisivas para la educación para la salud en la escuela secundaria

\subsection{Uso de películas y series en el ámbito académico}

En el análisis de la utilización de los materiales audiovisuales relacionados con las ciencias de la salud en las aulas, el $57,38 \%$ de los encuestados (136 estudiantes) responden que en sus institutos no se utilizan estos recursos. Sin embargo, el $42,62 \%$ reconoce que sí que se imparten clases utilizando films (101 de los encuestados). Dentro de los que responden que sí que se utilizan estos recursos, se valora la motivación y la finalidad con la que se utilizan en las clases (Gráfico 2).
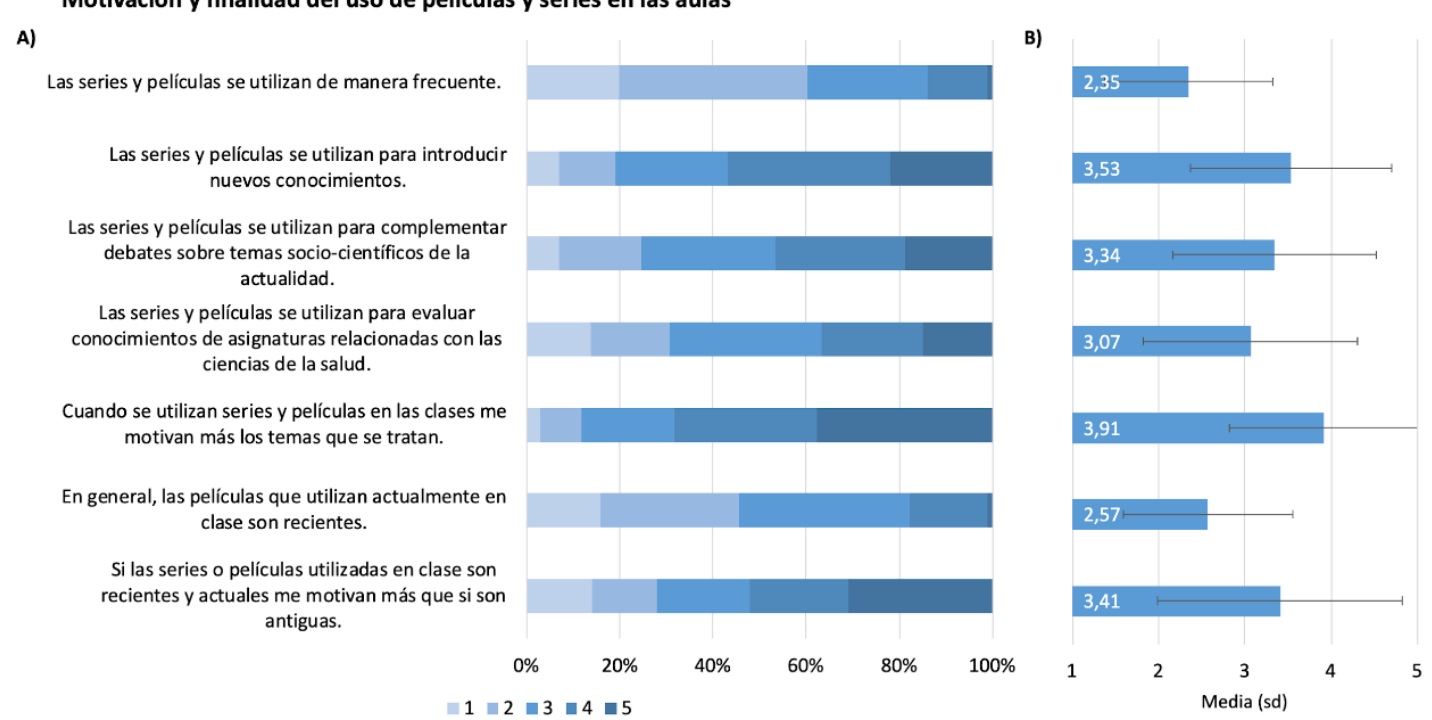

Gráfico 2. Motivación y finalidad del uso de películas y series en las aulas ( $N=101)$. 2A) porcentaje de menciones, 2B) media de las respuestas con la desviación estándar (sd) de cada ítem.

Fuente: elaboración propia

Cuando se pregunta si las películas y series se utilizan de manera frecuente en las aulas de educación secundaria, se obtiene una media de 2,35 sobre $5(\sigma=0,97)$ y el $60,40 \%$ de los encuestados están en desacuerdo o totalmente en desacuerdo con esta afirmación. Estos recursos se usan con diferentes finalidades docentes, la más común es para introducir nuevos conocimientos, con una media de 3,53 sobre $5(\sigma=1,17)$, en menor medida se usan para complementar debates socio-científicos de actualidad, con una media de 3,34 $(\sigma=1,18)$ y, en último lugar, se usan para evaluar conocimientos de asignaturas relacionadas con la salud, con una media de 3,07 $(\sigma=1,24)$. Sin embargo, parece que a menudo no se usan recursos de actualidad, obtenemos una media de 2,57 sobre $5(\sigma=0,98)$ cuando se pregunta si estos son recientes.

Por otro lado, el $68,32 \%$ de los estudiantes están de acuerdo o muy de acuerdo con el hecho de que cuando se utilizan estos recursos, les aumenta la motivación hacia los 
Interés y utilización de películas y series televisivas para la educación para la salud en la escuela secundaria

temas que se trabajan $(\square=3,91 ; \sigma=1,10)$, siendo el $37,62 \%$ los que están muy de acuerdo. Por último, obtenemos una media de 3,41 sobre $5(\sigma=1,41)$ si se pregunta si la motivación es mayor cuando estos materiales audiovisuales son recientes, con el $52 \%$ de los alumnos de acuerdo o muy de acuerdo con esta afirmación.

\subsection{Identificación de los objetivos de educación para la salud identificados en los materiales audiovisuales}

Nuevamente, se toman las respuestas de los 101 estudiantes que responden que, en las clases de secundaria, utilizar los recursos audiovisuales sí que se utilizan estos recursos en las clases de educación secundaria, y se analiza ahora la utilidad de estos materiales para la alfabetización en salud (Gráfico 3).

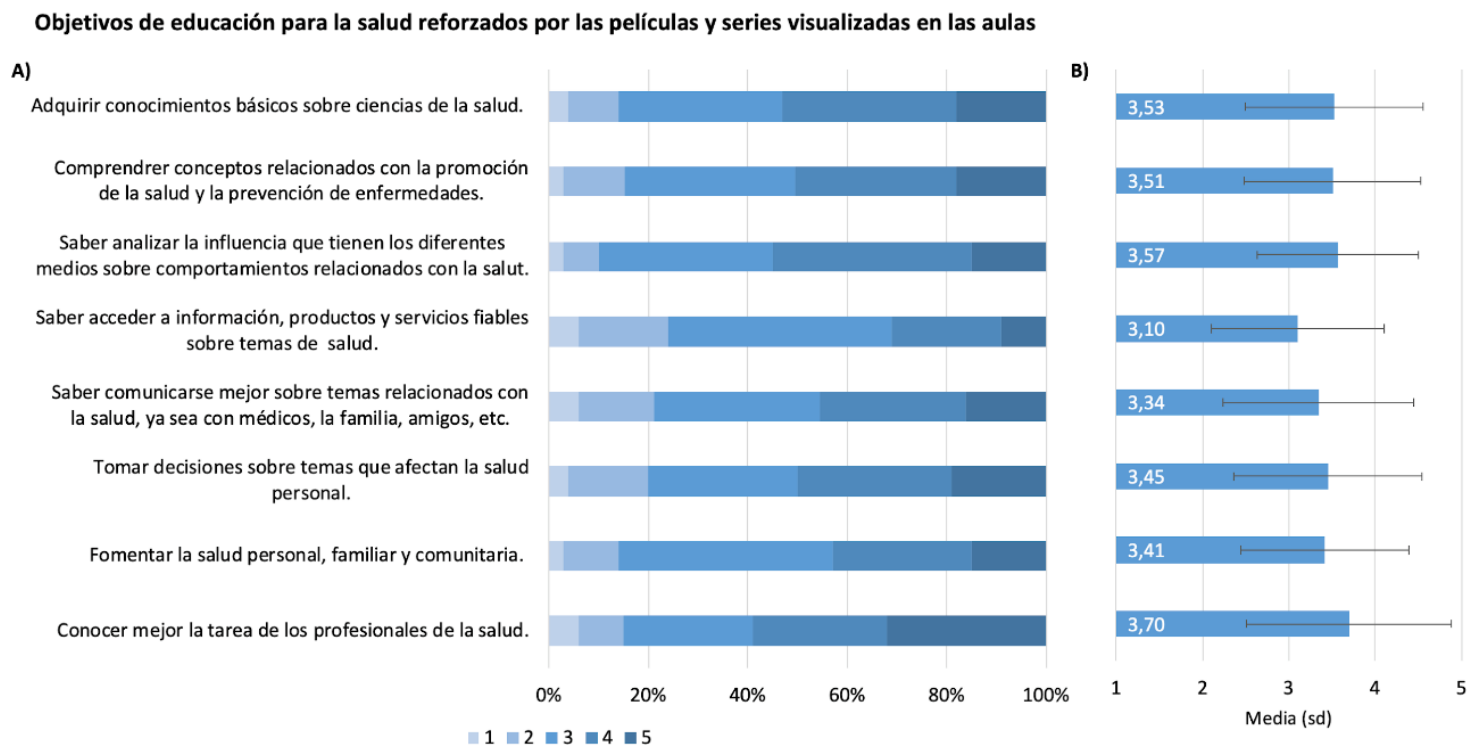

Gráfico 3. Objetivos de educación para la salud reforzados por las películas y series utilizadas en las aulas ( $N=101)$. 3A) porcentaje de menciones, 3B) media de las respuestas con la desviación estándar (sd) de cada ítem.

Fuente: elaboración propia

Cuando se pregunta si la utilización de estos recursos les ayuda a adquirir conocimientos básicos sobre ciencias de la salud, el 53\% muestra estar de acuerdo 0 muy de acuerdo $(\square=3,53 ; \sigma=1,03)$. De manera similar se obtiene una media de 3,51 sobre $5(\sigma=1,02)$ si se pregunta sobre la comprensión de conceptos relacionados con la promoción de la salud y la prevención de enfermedades. El 55\% de los estudiantes está de acuerdo o muy de acuerdo con que estas clases les ayuda a saber analizar la influencia que tienen la familia, los compañeros o los medios de comunicación sobre comportamientos relacionados con la salud $(\square=3,57 ; \sigma=0,93)$. Si nos centramos en el acceso a información fiable o bien en la comunicación sobre temas relacionados con la 
Interés y utilización de películas y series televisivas para la educación para la salud en la escuela secundaria

salud, se obtiene que no están ni de acuerdo ni en desacuerdo el $45 \%(\square=3,10 ; \sigma=1,00)$ y el $33,33 \%(\square=3,34 ; \sigma=1,11)$ respectivamente. Con respecto a cómo les han ayudado para la toma de decisiones sobre temas que afectan la salud, como por ejemplo frente a situaciones de consumo de drogas, toma de medicamentos o alimentación, obtenemos una media de 3,45 sobre $5(\sigma=1,10)$ y en el fomento de la salud personal, familiar y comunitaria una de 3,41 sobre $5(\sigma=0,98)$. Por último, el $59 \%(\square=3,70 ; \sigma=1,18)$, está de acuerdo o muy de acuerdo con que este tipo de clases les ayuda a conocer mejor la tarea que desarrollan los profesionales de la salud, siendo un $32 \%$ los que están muy de acuerdo.

\section{DISCUSIÓN Y CONCLUSIONES}

La educación para la salud comprende las oportunidades de aprendizaje para mejorar la alfabetización en salud e incluye la mejora del conocimiento de la población y el desarrollo de habilidades personales que conduzcan a la salud individual y comunitaria (Serrano Poveda, 2012). No solo se trata de transmitir información sino fomentar la motivación y las habilidades personales para adoptar medidas para cuidar la salud y prevenir enfermedades (Labonte, 1993).

Los medios audiovisuales constituyen una oportunidad innegable de transmitir información y fomentar la motivación y las habilidades personales. En esta investigación, encontramos como factores claves para establecer el uso de estos recursos en las aulas, el hecho de que las películas y series que miran los alumnos de manera autónoma muestren estos temas de salud y que les provoquen una motivación hacia este ámbito.

Como se puede observar en los resultados de este estudio exploratorio con estudiantes de educación secundaria de España, los materiales audiovisuales de ficción relacionados con las ciencias de la salud están bastante presentes en su vida cotidiana. Los estudiantes mencionan que miran series y películas relacionadas con las ciencias de la salud. Una gran variedad de materiales audiovisuales no son actuales pero pueden verse en distintas plataformas online como Netflix o HBO, que a través de sus catálogos ofrecen permanentemente productos de estreno pero también reposiciones de películas y series ya emitidas. La mayoría de los productos audiovisuales son de origen estadounidense, lo cual nos lleva a pensar que: por un lado, la industria cinematográfica norteamericana continúa siendo líder mundial en producción y distribución; y por otro lado, que la realidad de la sociedad, y en particular el sistema de salud, son diferentes según el contexto y esto permite explorar en clase distintas situaciones con implicaciones socioeconómicas.

Es interesante mencionar que los estudiantes recuerdan 35 series y 51 películas sobre temas de salud. Sin embargo, los materiales audiovisuales más visualizados son series médicas, lo cual coincide con la predominancia del tema de los hospitales.

Las películas y series más vistas están relacionadas con temáticas de hospitales. Los materiales audiovisuales más visualizados son las series médicas The Good Doctor y Grey's Anatomy. Esto coincide con los resultados de un estudio previo sobre el visionado

Revista de Comunicación y Salud, 2021, Vol. 11, 1-27 
Interés y utilización de películas y series televisivas para la educación para la salud en la escuela secundaria

de series médicas en estudiantes universitarios de ciencias de la salud, en mayor cantidad y frecuencia que las películas (Cambra-Badii et al., 2020). Estas series resultan relevantes ya que muestran la realidad que se viven en los hospitales y la de las múltiples situaciones que nos podemos encontrar en algún momento de nuestra vida donde la salud se puede ver afectada (Padilla, 2014).

The Good Doctor es una de las series médicas más nuevas, emitida desde 2017 en Estados Unidos (y a partir de 2018 en España), que muestra la vida de un médico joven que tiene autismo y síndrome de savant, y está comenzando su residencia en cirugía. La serie aborda los temas de salud desde un punto de vista diferente, con un protagonista que tiene marcadas dificultades en sus relaciones interpersonales y en la comunicación médico-paciente, y la originalidad de este personaje permite aumentar su interés por parte de los jóvenes (Cambra-Badii y Baños, 2018). Por otra parte, Grey's Anatomy mantiene su audiencia luego de 16 temporadas, y se emite semanalmente en los Estados Unidos y en España. En este caso, la serie médica se combina con el drama romántico y eso atrae a espectadores en todo el mundo (Weaver y Wilson, 2011; Czarny, 2008).

Además de películas y series que transcurren en hospitales, los estudiantes eligen ver materiales audiovisuales relacionados con la evolución, clonación e ingeniería genética. Esto es importante porque demuestra que los adolescentes también tienen visión sobre el mundo de las ciencias de la salud más allá de los hospitales. Estas películas permiten reflexionar sobre las posibles consecuencias de la investigación en clonación o ingeniería genética en un caso particular (como en la película Lucy) o como un instrumento socialmente aceptado (como en The Island) (Rodríguez y Baños, 2014).

En relación con la alta frecuencia de aparición de películas y menciones sobre epidemias y plagas, se puede entender que el interés por este tipo de enfermedades en particular puede reflejar el contexto en el cual se ha realizado el trabajo, en medio de la pandemia mundial de COVID-19. De hecho, en España se han multiplicado las descargas del film Contagion (2011) al inicio del confinamiento (El Mundo, 2020), y esta película retrata el desarrollo de una epidemia con varios puntos de similitud en relación con el coronavirus (Morales Vidal, 2020). En futuras investigaciones sobre este tema y otros puentes entre ficción y realidad, sería interesante poder corroborar si el contexto favorece que los estudiantes recuerden más estas películas, o si tienen más motivación por estudiar estos temas que reflejan su realidad cotidiana.

En cuanto a la mención de filmes que muestran temas sobre educación sexual y drogas, entendemos que son muy importantes para trabajar en la adolescencia. Los materiales audiovisuales nos abren puertas para trabajar estos temas de salud que pueden ser tabú pero muy necesarios de enseñar en el contexto de la educación secundaria (Ríos Espuny et al., 2018; Vega, 2002). En este caso, la función espontánea que el cine puede tener en la prevención y la contribución a la autonomía personal pueden ser recursos valiosos para indagar más puntualmente en otras investigaciones.

El segundo objetivo de la investigación, identificar qué objetivos de educación para la salud se tratan en las películas y series recordadas por los estudiantes, ha dado una

Revista de Comunicación y Salud, 2021, Vol. 11, 1-27 
Interés y utilización de películas y series televisivas para la educación para la salud en la escuela secundaria

amplia variedad de respuestas coincidentes en la utilidad de estos materiales audiovisuales.

Más de la mitad de los encuestados respondieron que están de acuerdo o muy de acuerdo con que estos materiales audiovisuales muestran conocimientos básicos sobre ciencias de la salud. El tema con el que hubo un mayor grado de acuerdo es la influencia que tienen las diferentes fuentes de información a la hora de establecer comportamientos saludables o bien en la toma de decisiones en situaciones que pueden comprometer la salud individual y comunitaria. Esto es importante, ya que, por ejemplo, ante una situación compleja, la decisión individual tomada puede ser clave a la hora de mantener la salud comunitaria (Kendal, 2019).

En segundo lugar, el tema que tuvo un amplio grado de acuerdo es la posibilidad de conocer mejor la tarea de los profesionales de la salud. Esta dimensión es importante porque permite conocer y discutir las representaciones profesionales en una visión realista, con los aciertos y errores profesionales cotidianos más allá de los clichés de locura o maldad, y por otro lado, porque permite explorar posibilidades de que los estudiantes desarrollen una vocación científica (García Sánchez, 2011).

Otros temas, como el acceso a información fiable o la comunicación sobre temas de salud con amigos y familiares, se manifiestan con mayor indiferencia que acuerdo: la mayoría de los estudiantes no está de acuerdo ni en desacuerdo con esas afirmaciones. Sin embargo, es necesario aclarar que hay aspectos que se pueden ver en las películas pero los estudiantes pueden no ser muy conscientes sobre esto, y por esto es necesario el aporte del profesor, tanto en el análisis de contenido previo del material audiovisual, la delimitación de objetivos de la clase, la conducción del debate, entre otras.

Se encuentran muy relevantes los resultados sobre el uso de las películas y series en las aulas de la escuela secundaria. Más de la mitad de los encuestados señalan que en sus institutos no se utilizan estos recursos, y dentro de los que responden que sí, la tendencia es que no se utiliza de manera frecuente. Sin embargo, cuando se identifican los objetivos para la educación para la salud que se trabajan en clase mediante materiales audiovisuales, los resultados son prácticamente similares a cuando se hace esta pregunta por los temas que identifican en la visualización de filmes que realizan de manera autónoma. Esto nos hace pensar que, efectivamente, estos recursos tienen un papel importante a la hora de trabajar los conceptos claves de salud. Resultaría interesante fomentar su uso en las aulas debido a que los estudiantes refieren más motivación hacia los temas que trabajan cuando se utilizan estos recursos, y la motivación es un factor clave para un correcto aprendizaje (Ospina Rodríguez, 2006).

Por otra parte, los estudiantes afirman que los motiva más si los materiales audiovisuales que se emplean son recientes, pero que efectivamente se utilizan más los que no lo son. ¿Cuáles pueden ser las razones para trabajar con películas y series que no sean de estreno reciente? Por un lado, hay materiales audiovisuales que sin dudas pueden considerarse "clásicos" por exponer temáticas de interés de una manera profunda 
Interés y utilización de películas y series televisivas para la educación para la salud en la escuela secundaria

y adecuada; pero por otro lado, se puede introducir la pregunta sobre la actualización de los materiales y las clases.

En relación con el modo de utilización de estos materiales audiovisuales, se encuentra que los estudiantes afirman que en las clases se utilizan más estos recursos para introducir nuevos conocimientos, en lugar de complementar debates socio-científicos actuales, lo cual enfatiza la importancia del material audiovisual y de la potencia que el cine y las series tienen como vehiculizadores conceptuales (Serra, 2011).

Es importante encontrar la manera ideal de trabajar estos conceptos en las aulas, poder llevar los materiales audiovisuales a un contexto real donde se interpreten situaciones que ellos mismos se puedan encontrar en su vida cotidiana y tener las herramientas para hacer frente a estas (Santesmases-Masana et al., 2017). Se pueden utilizar aspectos concretos de una película o una serie (tales como diálogos, secuencias narrativas, personajes), y diseñar actividades para re-pensar el material audiovisual en función de los objetivos educativos.

Siempre es necesario hacer una preparación previa donde se establezcan unos objetivos educativos a partir de lo que se quiere comunicar y hacer un buen diseño docente de selección del material audiovisual y del momento que se retransmitirá (Fresnadillo Martínez, 2005). Antes de la visualización del film es recomendable hacer una breve introducción en el aula de los objetivos que se trabajarán y a posteriori fomentar una discusión contextualizada, tanto con el profesor como con los compañeros, de los contenidos específicos para que los estudiantes sean capaces de comparar las diferentes situaciones mostradas con sus experiencias personales (Arroio, 2010; Kadivar et al., 2018). Asimismo, se pueden preparar actividades previas para que los estudiantes realicen una observación temática, y actividades para realizar de manera inmediata después de la visualización.

Podemos concluir que los resultados sugieren un uso favorable de los materiales audiovisuales actuales para trabajar con éxito los temas de alfabetización en salud a las clases de secundaria. Sin embargo, hay que fomentar más el uso de estos recursos para aprovechar el máximo rendimiento posible sobre la motivación de los estudiantes, el desarrollo de la empatía y las emociones, el trabajo a partir de un contexto real o verosímil, y la elaboración de contenidos relacionados con la toma de decisiones sobre la salud y valores, con un lenguaje diferente al habitual de las aulas y cercano a su vida cotidiana.

Para futuras investigaciones, interesa establecer estudios empíricos sobre la eficacia pedagógica del cine y las series (Cambra Badii y Baños, 2020) aplicados a escuelas de secundaria, así como también indagar sobre el consumo de cine y series y la utilización de estos materiales audiovisuales en clase en una muestra más amplia, representativa de toda España. Asimismo, resultaría interesante indagar sobre la utilización de estos materiales audiovisuales en las aulas de escuelas de secundaria desde la perspectiva de los docentes. La evaluación de los conocimientos a partir de la utilización de los materiales audiovisuales también debe ser estudiada en detalle. Por otra parte, se puede

Revista de Comunicación y Salud, 2021, Vol. 11, 1-27 
Interés y utilización de películas y series televisivas para la educación para la salud en la escuela secundaria

hacer un análisis profundo de los contenidos de las películas y series más mencionadas para poder identificar los temas concretos sobre salud que se tratan en cada una y poder definir unos objetivos docentes para trabajar en las aulas. Además se podría medir de manera práctica la alfabetización en salud entre adolescentes que se consigue objetivamente con estos recursos a través de instrumentos de medida cualitativa y cuantitativa como podría ser el HELMA ("Health Literacy Measure for Adolescentes") (Ghanbari et al., 2016) o pruebas más breves de reconocimiento de palabras como el REALM-Teen ("Rapid Estimate of Adolescente Literacy in Medicine") (Davis et al., 2006).

\section{REFERENCIAS}

Arroio, A. (2010). Context Based Learning: A Role for Cinema in Science Education. Science Education International, 21(3), 131-143. http://dx.doi.org/10.12691/education$\underline{5-2-6}$

Baños, J. E., y Pérez, J. (2005). Cómo fomentar las competencias transversales en los estudios de Ciencias de la Salud: una propuesta de actividades. Educación Médica, 8(4), 216-225. http://dx.doi.org/10.4321/S1575-18132005000500006

Baños, J. E., y Bosch, F. (2015). Using feature films as a teaching aid with medical students. Medical $\quad$ Teacher, 37(9), 883-884. http://dx.doi.org/10.3109/0142159x.2014.970997

Brigidi, S. (Eds.). (2016). Cultura, salud, cine y televisión. Recursos audiovisuales en ciencias de la salud y sociales. Tarragona, España: URV.

Bruselius-Jensen, M., Bonde, A. H. y Christensen, J. H. (2017). Promoting health literacy in the classroom. Health Education Journal, 76(2), 156-168. http://dx.doi.org/10.1177/0017896916653429

Cambra Badii, I., y Baños, J. E. (2020). The University goes to the movies: Our experience using feature films and tv series in teaching health sciences students. En S. Kim (Eds.), Medical Schools: Past, Present and Future Perspectives (pp. 105-148). New York: Nova Publishers.

Cambra Badii, I. y Baños, J. E. (2018). ¿Un médico autista en la televisión? Enseñanzas de The Good Doctor. Revista Medicina y Cine, 14(1), 57. https://revistas.usal.es/index.php/medicina y cine/article/view/19575

Cambra Badii, I. (2020). La utilización de las series televisivas en la enseñanza de las ciencias de la salud: nuevos aprendizajes y desafíos. Revista Medicina y Cine, 16(1), 57-58. http://dx.doi.org/10.14201/rmc20201615758

Cambra Badii, I., Guardiola, E. y Baños, J. E. (2020). From Marcus Welby, M.D. to The resident: the changing portrayal of physicians in TV medical dramas. Revista Medicina y Cine, 16(2), 87-102. http://dx.doi.org/10.14201/rmc202016287102 
Interés y utilización de películas y series televisivas para la educación para la salud en la escuela secundaria

Centers for Disease Control and Prevention (CDC) (2004). Joint Committee on National and Health Education Standards. National Health Education Standards. EEUU: CDC.

Chory-Assad, R. M. y Tamborini, R. (2001). Television doctors: An analysis of physicians on fictional and non-fictional television programs. Journal of Broadcasting and Electronic Media, 45(3), 499-552. http://dx.doi.org/10.1207/s15506878jobem4503 8

Correa Romero, F. E., Saldívar Garduño, A. y López Suarez, A. D. (2015). Autoconcepto Y Estados Emocionales: Su Relación Con La Motivación En Adolescentes. Enseñanza e Investigación en Psicología, 20(2), 173-183. http://dx.doi.org/10.21892/01239813.400

Creswell, J. W. (1998). Qualitative inquiry and research design: choosing among five traditions. California: Sage Publications.

Czarny, M. J., Faden, R. R., Nolan, M. T., Bodensiek, E. y Sugarman, J. (2008). Medical and nursing students' television viewing habits: potential implications for bioethics. The $\begin{array}{llll}\text { American Journal of Biothics, } & \text { 8(12), }\end{array}$ http://dx.doi.org/10.1080/15265160802595074

D’Ottavio, A., García Sánchez, J. E. y García Sánchez, E. (2019). The cinema as a fostering tool for health area undergraduates. Revista Medicina y Cine, 15(2), 101-105. http://dx.doi.org/10.14201/rmc2019152101105

Davis, T. C., Wolf, M. S., Arnold, C. L., Byrd, R. S., Long, S. W., Springer, T. y Bocchini, J. A. (2006). Development and validation of the Rapid Estimate of Adolescent Literacy in Medicine (REALM-Teen): A tool to screen adolescents for below-grade reading in health care settings. Pediatrics, 118(6), 1707-1714. http://dx.doi.org/10.1542/peds.2006-1139

De Lellis, M., Calzetta, C. y Gómez, T. (2014). Promoción de la salud en entornos educativos. El empleo de NTIC en el aprendizaje en salud. Revista Iberoamericana de Educación, 66, 175-188. http://dx.doi.org/10.35362/rie660386

El Mundo (10 de marzo de 2020). El coronavirus hace que se disparen las descargas de esta película de 2011 que no está en Netflix. El Mundo, sección Tecnología. https://www.elmundo.es/tecnologia/2020/03/10/5e6660eefc6c8307608b45ea.html

Falcón Romero, M. y Ruiz-Cabello A. L. (2012). Alfabetización en salud; concepto y dimensiones. Proyecto europeo de alfabetización en salud. Revista Comunicación y Salud, 2(2), 91-98. http://dx.doi.org/10.35669/revistadecomunicacionysalud.2012.2(2).91-98

Farré, M. (2013). Señores docentes, más cine por favor. Revista Medicina y Cine, 9(2), 51-52. http://dx.doi.org/10.14201 
Interés y utilización de películas y series televisivas para la educación para la salud en la escuela secundaria

Fresnadillo Martínez, M. J., Diego Amado, M. C., García Sánchez, E. y García Sánchez, J. E. (2005). Metodología docente para la utilización del cine en la enseñanza de la microbiología médica y las enfermedades infecciosas. Revista Medicina y Cine. 1(1), 17-23. https://revistas.usal.es/index.php/medicina y cine/article/view/228

García-Borrás, F. J. (2009). Bienvenido Mister Cine a la enseñanza de las ciencias. Revista Eureka sobre Enseñanza y Divulgación de las Ciencias, 6(1), 79-91. https://rodin.uca.es/xmlui/handle/10498/10300

Gavidia Catalán, V., Garzón Fernández, A., Talavera Ortega, M., Sendra Mocholí, C. y Mayoral García-Berlanga, O. (2019). Alfabetización en salud a través de las competencias. Enseñanza las Ciencias Revista Investigación y Experiencias didácticas, 37(2), 107-126. http://dx.doi.org/10.5565/rev/ensciencias.2628

Ghanbari, S., Ramezankhani, A., Montazeri, A. y Mehrabi, Y. (2016). Health literacy measure for adolescents (HELMA): Development and psychometric properties. Public Library of Science One, 11(2), 1-12. http://dx.doi.org/10.1371/journal.pone.0149202

Gobierno de España-Ministerio de Educación y Ministerio de Sanidad-Política Social e Igualdad (2009). Ganar Salud en la Escuela. Guía para conseguirlo. Madrid, España: Ministerio de Educación y Ministerio de Sanidad.

Icart-Isern, M. T., Garrido-Aguilar, E., y Miguel-Cañas, S. (2013). Formación en enfermería escolar: efectividad del taller 'Cine y salud escolar'. FEM: Revista de la Fundación Educación Médica, 16(4), 239-244. http://dx.doi.org/10.4321/S2014$\underline{98322013000600009}$

Kadivar, M., Mafinejad, M. K., Bazzaz, J. T., Mirzazadeh, A., y Jannat, Z. (2018). Cinemedicine: Using movies to improve students' understanding of psychosocial aspects of medicine. Annals on Medicine and Surgery, 28(28), 23-27. http://dx.doi.org/10.1016/i.amsu.2018.02.005

Kendal, E. (2019). Public health crises in popular media: How viral outbreak films affect the public's health literacy. Medical Humanities. http://dx.doi.org/10.1136/medhum2018-011446

Kirby, D. A. (2017). The Changing Popular images of Science. En K. H. Jamieson, D. Kahan y D. A. Scheufele, The oxford handbook of the science of science communication, USA, Oxford library of psychology, 291-298. http://dx.doi.org/10.1093/oxfordhb/9780190497620.001.0001

Krippendorf, K. (1997). Metodología de análisis de contenido: Teoría y práctica. Barcelona, España: Paidós. 
Interés y utilización de películas y series televisivas para la educación para la salud en la escuela secundaria

Labonte, R. (1993). Health promotion and empowerment: Practice frameworks. Toronto: Centre for Health Promotion and Participation. http://dx.doi.org/10.1177/109019819402100209

Law, M., Kwong, W., Friesen, F., Veinot, P. y Ng, S. (2015). The current landscape of television and movies in medical education. Perspectives on Medical Education, 4, 218-224.

Lobato, R. (2018). Rethinking international TV flows research in the age of Netflix. Television \& New Media, 19(3), 241-256.http://dx.doi.org/10.1007/s40037-015-0235-3

Machado, M. L., Oliveira, D. L. y Manica, S. T. (2013). Extended nursing consultation: education possibilities for the practice of integrality in health. Revista Gaúcha de Enfermagem, 34(4), 53-60. http://dx.doi.org/10.1590/s1983-14472013000400007

Manganello, J, A. (2008). Health literacy and adolescents: A framework and agenda for future research. Health Education Research, 23(5), 840-847. http://dx.doi.org/10.1093/her/cym069

Mateus, J. C. (2017). Las teleseries también educan. Una defensa de las ficciones televisivas como dispositivos de aprendizaje. En G. Cappello, Ficciones cercanas. Televisión, narración y espíritu de los tiempos (pp. 179-195). Lima, Perú: Universidad de Lima.

McMillan, J. H. y Schumacher, S. (2005) Investigación Educativa. Una introducción conceptual. Madrid: Pearson Addison Wesley.

Morales Vidal, P. (2020). COVID-19, cuando la ficción se convierte en realidad. Dr. Antoni Esteve Fundació. https://cutt.ly/YkaCbsg

Nutbeam, D., McGill, B., y Premkumar, P. (2018). Improving health literacy in community populations: A review of progress. Health Promotion International, 33(5), 901-911. http://dx.doi.org/10.1093/heapro/dax015

Nutbeam, D. (2000). Health literacy as a public health goal: a challenge for contemporary health education and communication strategies into the 21 st century. Health Promotion International, 15(3), 259-267. http://dx.doi.org/10.1093/heapro/dax015

O'Connor, M. M. (1998). The role of the television drama ER in medical student life: $\begin{array}{llll}\text { entertainment } & \text { or socialization? JAMA, 280(9), 854-855. }\end{array}$ http://dx.doi.org/10.1001/jama.280.9.854

Organización Mundial de la Salud (OMS) (1990). Carta de Ottawa para la Promoción de la salud. Conferencia Internacional auspiciada por la OMS y la Asociación Canadiense de Salud Pública. Toronto, Canadá: OMS. 
Interés y utilización de películas y series televisivas para la educación para la salud en la escuela secundaria

Organización Mundial de la Salud (OMS) (1986). Primera Conferencia Internacional sobre Fomento de la Salud. Carta de Ottawa para la promoción la salud. Toronto, Canadá: OMS.

Ospina Rodríguez, J. (2006). La motivación, motor del aprendizaje. Revista ciencias de la salud, 4(2), 158-160. https://www.redalyc.org/articulo.oa?id=56209917

Padilla, G. (2014). La influencia de las series de médicos en los espectadores y en los profesionales de la salud. SAPIENS, 15(1), 51-68.

Petit, M. F. y Solbes, J. (2012). La ciencia ficción y la enseñanza de las ciencias. Enseñanza de las Ciencias, 30(2), 55-72. https://www.raco.cat/index.php/Ensenanza/article/view/254503

Petit, M. F. y Solbes, J. (2015). El cine de ciencia ficción en las clases de ciencias de enseñanza secundaria (I). Propuesta didáctica. Revista Eureka sobre Enseñanza y Divulgación de las Ciencias, 12(2), 311-327. http://dx.doi.org/0.25267/Rev Eureka ensen divulg cienc.2015.v12.i2.06

Petit, M. F. y Solbes, J. (2016). El cine de ciencia ficción en las clases de ciencias de enseñanza secundaria (II). Análisis de películas. Revista Eureka sobre Enseñanza y Divulgación de las Ciencias, 13(1), 176-191. http://hdl.handle.net/10498/18022

Real Decreto 126/2014, de 28 de febrero, de Educación Primaria. Boletín Oficial del Estado 52, Gobierno de España, 1 de marzo de 2014. Recuperado el 31 de agosto de 2020 de: https://www.boe.es/buscar/pdf/2014/BOE-A-2014-2222-consolidado.pdf

Real Decreto 1105/2014, de 26 de diciembre, de Educación Secundaria Obligatoria y del Bachillerato. Boletín Oficial del Estado 3, Gobierno de España, 3 de enero de 2015. Recuperado el 31 de agosto de 2020 de: https://www.boe.es/boe/dias/2015/01/03/pdfs/BOE-A-2015-37.pdf

Ríos Espuny, A. F., Bataller i Perelló, V., Tárraga, P. J., Casanova, A. y Bonilla, A. (2018). Salud Sexual: La gran olvidada del ámbito educativo. Journal of Negative and No Positive Results, 3(12), 946-957. http://dx.doi.org/10.19230/jonnpr.2641

Rodríguez Torres, Á. F., Páez Granja, R. E., Altamirano Vaca, E. J., Paguay Chávez, F. W., Rodríguez Alvear, J. C., y Calero Morales, S. (2017). Nuevas perspectivas educativas orientadas a la promoción de la salud. Educación Médica Superior, 31(4), 1-11. http://www.ems.sld.cu/index.php/ems/article/view/1366

Rodríguez, G., Baños, J. E. (2014). Réplicas y perfección humana: Los niños del Brasil y Gattaca. Revista Medicina y Cine, 10(2), 60-67. https://revistas.usal.es/index.php/medicina y cine/article/view/13570 
Interés y utilización de películas y series televisivas para la educación para la salud en la escuela secundaria

Santesmases-Masana, R., González-de Paz, L., Real, J., Borràs-Santos, A., SisóAlmirall, A. y Navarro-Rubio, M. D. (2017). Alfabetización en salud en pacientes con insuficiencia cardiaca atendidos en atención primaria. Atención Primaria, 49(1), $28-34$. http://dx.doi.org/10.1016/j.aprim.2016.03.003

Serra, M. S. (2011). Cine, escuela y discurso pedagógico: Articulaciones, inclusiones y objeciones en el siglo XX en Argentina. Buenos Aires, Argentina: Teseo.

Serrano Poveda, M. E. (2012). Introducción en educación para la salud: fundamentos, claves y conceptos básicos. Formación Activa en Pediatría de Atención Primaria, 5(4), 246-257.

Sørensen, K., Van Den Broucke, S., Fullam, J., Doyle, G., Pelikan, J., Slonska, Z. y Consortium Health Literacy Project European (2012). Health literacy and public health: A systematic review and integration of definitions and models. BMC Public Health, 12(80), 1-13. http://dx.doi.org/10.1186/1471-2458-12-80

Tapper, E. B. (2010). Doctors on Display: The Evolution of Television's Doctors. Baylor University Medical Center Proceedings, 23(4), 393-399. http://dx.doi.org/10.1080/08998280.2010.11928659

Úbeda Carulla, J. (2016). Cámaras, informes y hospitales. En S. Brigidi (Eds.), Cultura, salud, cine y televisión. Recursos audiovisuales en ciencias de la salud y sociales (pp. 23-32). Tarragona, España: URV. http://dx.doi.org/10.17345/9788484244233

Vasilachis de Gialdino, I. (2006) (coord) Estrategias de Investigación Cualitativa. Barcelona: Gedisa.

Vega, A. (2002). Cine, drogas y salud: recursos para la acción educativa. Comunicar: Revista Científica de Comunicación y Educación, 9(18), 123-129. https://www.revistacomunicar.com/ojs/index.php/comunicar/article/view/C18-2002-24

Wager, J., Stahlschmidt, L., Heuer, F., Troche, S. y Zernikow, B. (2018). The impact of a short educational movie on promoting chronic pain health literacy in school: A feasibility study. European Journal of Pain, 22(6), 1142-1150. http://dx.doi.org/10.1002/ejp.1202

Weaver, R. y Wilson, I. (2011). Australian medical students' perceptions of professionalism and ethics in medical television programs. BMC Medical Education, 11(50). http://dx.doi.org/10.1186/1472-6920-11-50

Williams, D. J., Re, D. y Ozakinci, G. (2014). Television viewing habits of preclinical UK medical undergraduates: further potential implications for bioethics. AJOB Empirical Bioethics, 5(2), 55-67. http://dx.doi.org/10.1080/21507716.2013.826297

\section{AUTORES}


Interés y utilización de películas y series televisivas para la educación para la salud en la escuela secundaria

\section{Carla Garrido Pérez}

Graduada en Biología Humana, Universitat Pompeu Fabra, Barcelona, España. Cursando el Máster en Formación del profesorado de ESO y Bachillerato, Formación profesional y enseñanza de idiomas (UdG). Interesada en la educación para las ciencias de la salud y la innovación en métodos de aprendizaje. En esta línea, ha realizado el Trabajo de Fin de Grado sobre el uso de películas y series en las aulas para promover la alfabetización en salud de los estudiantes.

Orcid ID: https://orcid.org/0000-0002-7563-0467

\section{Irene Cambra Badii}

Investigadora en la cátedra de Bioética de la Universitat de Vic - Universitat Central de Catalunya y en el Grupo de Investigación en Educación en Ciencias de la Salud (GRECS) en el Departamento de Ciencias Experimentales y de la Salud de la Universitat Pompeu Fabra, Barcelona, España. Trabaja temas de investigación ligados con la utilidad del cine y las series para la enseñanza de las ciencias de la salud, particularmente de la bioética. Orcid ID: https://orcid.org/0000-0003-1233-3243

\section{Mar Carrió Llach}

Investigadora del Grupo de Investigación Educativa en Ciencias de la Salud (GRECS), en el Departamento de Ciencias Experimentales y de la Salud de la Universitat Pompeu Fabra, Barcelona, España. Sus principales líneas de investigación se focalizan en el aprendizaje basado en problemas y en la indagación en la docencia biomédica y el fomento de las competencias de creatividad y pensamiento crítico. Ha publicado numerosos artículos y capítulos de libro en el ámbito de la educación en las ciencias de la salud y participado en varios proyectos nacionales e internacionales.

Orcid ID: https://orcid.org/0000-0001-5585-7288 
Interés y utilización de películas y series televisivas para la educación para la salud en la escuela secundaria

\section{Anexo}

\section{Cuestionario}

Título: Estudio del uso del cine y series en las aulas para la educación de las ciencias de la salud

Descripción:

Si estás estudiando la ESO o Bachillerato, ¿serias tan amable de contestar esta encuesta? No os llevará más de 5-8 minutos.

Este cuestionario es parte de mi trabajo de fin de grado de la carrera de Biología Humana (UPF). Mediante este estudio me propongo conocer qué impacto tienen las películas y series que visualizáis sobre la educación de ciencias de la salud, además de saber qué uso se hace actualmente del cine comercial y series en las clases de secundaria y bachillerato.

Muchas gracias por la participación y la ayuda en realizar el estudio.

Dimensión 1:

- Género: Mujer, Hombre, Otros

- Curso actual (1ํㅡㄴ -2 Bachillerato)

- Comunidad Autónoma en la que estudias

Dimensión 2: Impacto de películas y series en la vida cotidiana

- ¿Sueles visualizar películas o series relacionadas con el ámbito de las ciencias de la salud (sector hospitalario, sobre enfermedades, sobre laboratorios, mutaciones, evolución, genética...)? Sí/NO

- Cita las películas o series que recuerdes haber visto recientemente sobre el ámbito de las ciencias de la salud (sector hospitalario, enfermedades, laboratorios, mutaciones, evolución, genética...).

Puntúa del 1 (totalmente en desacuerdo) al 5 (muy de acuerdo) las siguientes afirmaciones sobre el impacto que tienen las series y películas en el aprendizaje informal de las ciencias de la salud.

- Considero que estas series y películas me han motivado a querer saber más sobre temas relacionados con las ciencias de la salud.

En estas series y películas se tratan temas como:

- Conocimientos básicos sobre ciencias de la salud (mutaciones, ingeniería genética, ADN, enfermedades hereditarias, clonación, etc.)

- Conceptos relacionados con la promoción de la salud y la prevención de enfermedades (relación entre conductas saludables y salud personal, comparación y contraste de ventajas y desventajas de mantener prácticas saludables...).

- La influencia que tienen la familia, los compañeros, los medios de comunicación o la tecnología, sobre comportamientos relacionados con la salud.

- El acceso a información, productos y servicios fiables sobre temas de salud.

- La comunicación sobre temas relacionados con la salud ya sea con médicos, la familia, amigos, etc. 
Interés y utilización de películas y series televisivas para la educación para la salud en la escuela secundaria

- La toma de decisiones que afectan a la salud personal delante de situaciones como el consumo de drogas, la alimentación, el uso de diferentes tratamientos médicos, etc.

- Aspectos de responsabilidad sobre la salud personal, familiar y comunitaria (contagios de enfermedades infecciosas, vacunación, etc.).

- Las tareas que desarrollan los profesionales de la salud (médicos, enfermeros, investigadores, etc.)

Después de leer las cuestiones, ¿queréis añadir alguna película o serie de la que os hayáis acordado que traten los temas anteriormente expuestos?

\section{Dimensión 3: Uso de películas y series en el ámbito académico}

- ¿En tu instituto utilizan películas comerciales y series para realizar clases relacionadas con ciencias de la salud? Sí/NO

- Si la respuesta anterior es afirmativa, responde las siguientes cuestiones valoradas en una escala del 1 (totalmente en desacuerdo) al 5 (muy de acuerdo).

- Las series y películas se utilizan de manera frecuente.

- Las series y películas se utilizan para introducir nuevos conocimientos.

- Las series y películas se utilizan para complementar los debates sobre temas socio-científicos de actualidad.

- Las series y películas se utilizan para evaluar conocimientos de asignaturas relacionadas con ciencias de la salud.

- Cuando se utilizan series y películas en las clases me motivan más los temas que se tratan.

- Por lo general, las películas que utilizan actualmente en clase son recientes.

- Si las series o películas utilizadas en clase son recientes y actuales me motivan más que si son antiguas.

La visualización de estas series y películas en el aula, me han ayudado a:

- Adquirir conocimientos básicos sobre ciencias de la salud (mutaciones, ingeniería genética, ADN, enfermedades hereditarias, clonación, etc.)

- Comprender conceptos relacionados con la promoción de la salud y la prevención de enfermedades (relación entre conductas saludables y salud personal, comparación y contraste de ventajas y desventajas de mantener prácticas saludables...).

- Saber analizar la influencia que tienen la familia, los compañeros, los medios de comunicación o la tecnología, sobre comportamientos relacionados con la salud.

- Saber acceder a información, productos y servicios fiables sobre temas de salud.

- Saber comunicarme mejor sobre temas relacionados con la salud, ya sea con médicos, la familia, amigos, etc.

- Tomar decisiones sobre temas que afectan a la salud personal, en situaciones como el consumo de drogas, la alimentación, el uso de diferentes tratamientos médicos, etc.

- Fomentar la salud personal, familiar y comunitaria.

- Conocer mejor la labor de los profesionales de la salud (médicos, enfermeros, investigadores, etc.) 